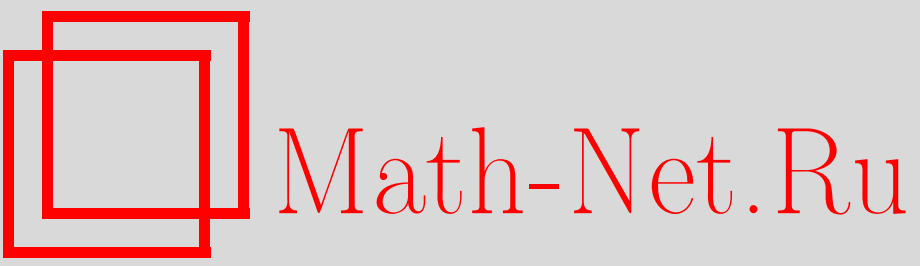

Ю. И. Лютахин, Экстремумы вектор-функции индукции вращательносимметричного магнитного поля стационарного контурного тока в "сферическом" многосредном пространстве, Вестн. Сам. гос. техн. ун-та. Сер. Физ.мат. науки, 2007, выпуск 2(), 116-122

DOI: https://doi.org/10.14498/vsgtu539

Использование Общероссийского математического портала Math-Net.Ru подразумевает, что вы прочитали и согласны с пользовательским соглашением http: //www . mathnet.ru/rus/agreement

Параметры загрузки:

IP : 3.85 .183 .62

26 апреля 2023 г., 13:36:34 
Вестн. Сам. гос. техн. ун-та. Сер.: Физ.-мат. науки. - 2007. - № 2(15). - С. 116-122. - ISSN $1991-8615$

УДК 658.011.054

Ю. И. Лютахин

\section{ЭКСТРЕМУМЫ ВЕКТОР-ФУНКЦИИ ИНДУКЦИИ ВРАЩАТЕЛЬНО-СИММЕТРИЧНОГО МАГНИТНОГО ПОЛЯ СТАЦИОНАРНОГО КОНТУРНОГО ТОКА В «СФЕРИЧЕСКОМ» МНОГОСРЕДНОМ ПРОСТРАНСТВЕ}

Предлагается численно-аналитический метод расчёта экстремальных значений индукиии магнитного поля стационарного кругового контурного тока в сфферическом пространстве магнитно-разнородных сред. Для случая экваториального расположения витка с током условия экстремума вектор-фбункиии индукции доведены до законченного аналитического решения в явных бункииях.

Особенности функционирования, а также большое разнообразие конструкций электромагнитных систем со сферической геометрией, применяемых в электроприводах сканирующих, гироскопических, навигационных и робототехнических устройств; в установках, обеспечивающих электромагнитную совместимость режимов работы электрооборудования, требуют создания адекватных математических моделей, малозатратных методов проектирования и методик расчёта.

Существующие модели [1-13] дают полезную информацию для решения конкретных задач, однако они не учитывают возможности произвольного варьирования числа сред; накладывают ограничения на значения магнитных проницаемостей материалов сред; не позволяют определять экстремальные значения индукции магнитного поля, характеризующие магнитный режим работы магнитопровода электромагнитной системы. Нет опубликованных работ по методике инженерного расчёта и проектирования аналогичных систем.

Характер и поведение поля электромагнитной системы полностью определяет её качественные свойства и количественные характеристики, параметры. По этой причине одним из эффективных направлений исследований является математическое моделирование электромагнитных полей с доведением конечного результата до выражений в явных функциях и последующее создание САПР. При этом, в силу сложности электромагнитных процессов, целесообразным является использование при проектировании моделей магнитных полей стационарных токов с последующим уточнением на основе теоретической и экспериментальной коррекции методик расчёта.

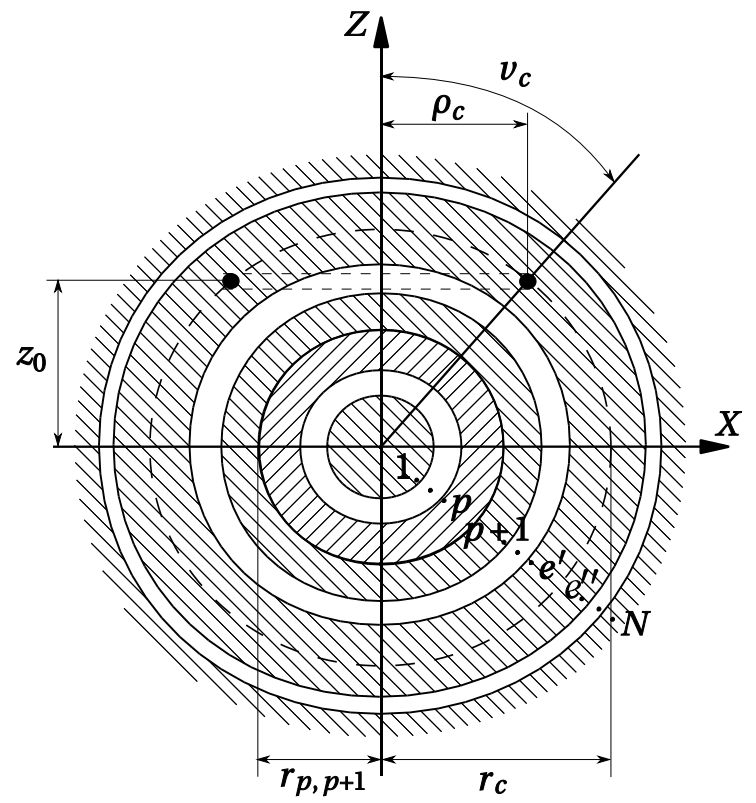

Электромагнитная система

С этой целью целесообразным является использование классического исследования вектор-фрункций, описывающих электромагнитное поле на максимум и минимум [14-17].

Для облегчения решения поставленной краевой задачи, представим реальную электромагнитную систему в виде модели: круговой, бесконечно тонкий виток со стационарным током осесимметрично расположен в одной из областей пространства, разделённого софокусными, сферическими поверхностями на произвольное количество областей, занятых магнитноразнородными изотропными средами (см. рис.). Поле же, созданное током реальной катушки с конечным поперечным сечением, определяется суперпозицией полей совокупности бесконечно тонких витков $[12,16,18]$.

На основании теории классической электродинамики и решения краевой задачи, поле в любой точке области определяется суперпозицией полей возбуждения и искажения. Векторный магнитный потенциал - из выражения

$$
\vec{A}^{(p)}=\vec{A}^{(p)^{\prime}}+\vec{A}^{(p)^{\prime \prime}} .
$$

Индукция магнитного поля - из выражения

$$
\vec{B}^{(p)}=\operatorname{rot} \vec{A}^{(p)} .
$$


С учётом вращательно-симметричной геометрии имеем

$$
\vec{A}^{(p)}=A_{\varphi}^{(p)} \vec{i}_{\varphi} ; \quad \overrightarrow{B^{(p)}}=\vec{i}_{r} B_{r}^{(p)}+\vec{i}_{\theta} B_{\theta}^{(p)},
$$

где $\vec{i}_{r}, \vec{i}_{\theta}, \vec{i}_{\varphi}$ - ортогональные орты сорерической системы координат; $p$ - индекс среды.

Векторный потенциал поля возбуждения бесконечно-тонкого кругового витка в однородном и изотропном пространстве с учётом вращательно-симметричной геометрии определяется из выражения [1, 19]:

$$
\vec{A}=\vec{i}_{\varphi} \frac{\mu_{p} I a^{2} r \sin \theta}{\left(z_{0}^{2}+r^{2}-2 z_{0} r \cos \theta+a^{2}\right)^{3 / 2}} \sum_{n=0}^{\infty} \prod_{i=0}^{2 n} \frac{\frac{1}{2}+i}{1+i} \prod_{j=1}^{n+1} \frac{2 j-1}{2 j}\left(\frac{2 a r \sin \theta}{z_{0}^{2}+r^{2}-2 z_{0} r \cos \theta+a^{2}}\right)^{2 n},
$$

где $\mu_{p}$ - абсолютная магнитная проницаемость $p$-среды магнитной системы, $\Gamma_{\text {н }} / \mathrm{m} ; a=r_{c}-$ радиус окружности контура тока, м; I-величина тока в витке, А; $z_{0}-$ сдвиг плоскости витка вдоль оси $\theta=0 ; p=0,1, \ldots, N-$ индекс среды системы; $n=0,1, \ldots, \infty ; i=0,2, \ldots, 2 n ; j=1,2, \ldots, n+1-$ немые индексы; $r, \theta, \varphi$ - координаты сферической системы. Область определения фрункции (4) все бесконечное пространство $r \geqslant 0 ; 0 \leqslant \theta \leqslant \pi ; 0 \leqslant \varphi \leqslant 2 \pi$, за исключением контура тока.

Компоненты вектора индукции $\vec{B}^{(p)^{\prime}}$ неискажённого магнитного поля витка с током в $p$-среде пространства с учётом выражения (2) определяются следующим образом:

$$
\begin{aligned}
& B_{r}^{(p)^{\prime}}(r ; \theta)=\frac{2 \mu_{p} I a^{2}\left(r \cos \theta-z_{0}\right)}{\left(z_{0}^{2}+r^{2}-2 z_{0} r \cos \theta+a^{2}\right)^{3 / 2}\left(z_{0}^{2}+r^{2}-2 z_{0} r \cos \theta\right)^{1 / 2}} \sum_{n=0}^{\infty} \prod_{i=1}^{2 n} \frac{\frac{1}{2}+i}{1+i} \times \\
& \times \prod_{j=1}^{n+1} \frac{2 j-1}{2 j}\left(\frac{2 a r \sin \theta}{z_{0}^{2}+r^{2}-2 z_{0} r \cos \theta+a^{2}}\right)^{2 n}(n+1), \\
& B_{\theta}^{(p)^{\prime}}(r ; \theta)=\frac{-\mu_{p} I a^{2} r \sin \theta}{\left(z_{0}^{2}+r^{2}-2 z_{0} r \cos \theta+a^{2}\right)^{3 / 2}\left(z_{0}^{2}+r^{2}-2 z_{0} r \cos \theta\right)^{1 / 2}} \sum_{n=0}^{\infty} \prod_{i=1}^{2 n} \frac{\frac{1}{2}+i}{1+i} \times \\
& \times \frac{2(n+1) a^{2}-\left(z_{0}^{2}+r^{2}-2 z_{0} r \cos \theta\right)(2 n+1)}{z_{0}^{2}+r^{2}-2 z_{0} r \cos \theta+a^{2}} \prod_{j=1}^{n+1} \frac{2 j-1}{2 j}\left(\frac{2 a r \sin \theta}{z_{0}^{2}+r^{2}-2 z_{0} r \cos \theta+a^{2}}\right)^{2 n} .
\end{aligned}
$$

Область определения фрункций (5) и (6) та же, что и для фрункции (4).

Согласно принципу наложения или суперпозиции неискажённого поля и поля искажения $[1,12,16,18]$, векторный потенциал поля искажения обусловленного, лишь неоднородностью пространства, является решением уравнения Лапласа

$$
\Delta \vec{A}^{(p)^{\prime \prime}}=0
$$

В сорерической системе координат, во вращательно-симметричном случае, система трёх скалярных уравнений Лапласа, соответствующая векторному уравнению (7), эквивалентна одному скалярному диффреренциальному уравнению второго порядка в частных производных относительно одной неизвестной ( $\varphi$ - компоненты векторного потенциала), а остальные его компоненты $A_{r}^{(p)^{\prime \prime}}=A_{\theta}^{(p)^{\prime \prime}}=0$ :

$$
\frac{\partial}{\partial r}\left(r^{2} \frac{\partial A_{\varphi}^{(p)^{\prime \prime}}}{\partial r}\right)+\frac{1}{\sin \theta} \frac{\partial}{\partial \theta}\left(\sin \theta \frac{\partial A_{\varphi}^{(p)^{\prime \prime}}}{\partial \theta}\right)-\frac{A_{\varphi}^{(p)^{\prime \prime}}}{\sin ^{2} \theta}=0
$$

С использованием метода разделения переменных, общее решение уравнения (8) представляется комбинацией его частных решений вида

$$
A_{\varphi}^{(p)^{\prime \prime}}(r ; \theta)=\sum_{n=0}^{\infty}\left(C_{n+1}^{(p)} r^{n+1}+D_{n+1}^{(p)} r^{-n-2}\right) P_{n+1}^{1}(\cos \theta)
$$


Компоненты индукции магнитного поля искажения в $p$-среде определяются, с учётом формулы (2), из выражений

$$
\begin{aligned}
& B_{r}^{(p)^{\prime \prime}}(r ; \theta)=\sum_{n=0}^{\infty}\left(C_{n+1}^{(p)} r^{n}+D_{n+1}^{(p)} r^{-n-3}\right)\left[P_{n+1}^{1}(\cos \theta)-P_{n-1}^{1}(\cos \theta)\right] \\
& B_{\theta}^{(p)^{\prime \prime}}(r ; \theta)=-\sum_{n=0}^{\infty}\left(C_{n+1}^{(p)} r^{n}(n+2)-D_{n+1}^{(p)} r^{-n-3}(n+1)\right) P_{n+1}^{1}(\cos \theta)
\end{aligned}
$$

где $C_{n}^{(p)}$ и $D_{n}^{(p)}$ - постоянные коэффициенты интегрирования, определяемые для каждой среды системы из решения краевой задачи преломления магнитного поля; $P_{n+1}^{1}(\cos \theta)-$ присоединённые функции Лежандра первого рода. Из условия инвариантности тангенциальной составляющей напряжённости $\vec{H}$ магнитного поля при переходе через границу раздела двух сред $p$ и $p+1$ $[17,19]$ имеем

$$
H_{\theta}^{(p)}\left(r_{p, p+1} ; \theta\right)=H_{\theta}^{(p+1)}\left(r_{p, p+1} ; \theta\right),
$$

где $r_{p, p+1}$ - радиус граничной сфреры. С учётом $\vec{B}^{(p)}=\mu_{p} \vec{H}^{(p)}$, из выражений (2), (3), (6) и (11) получается первая бесконечная подсистема алгебраических уравнений, содержащая $n(N-1)$ уравнений:

$$
\mu_{p}^{-1}\left(C_{n+1}^{(p)}(n+2) r_{p, p+1}^{n}-D_{n+1}^{(p)}(n+1) r_{p, p+1}^{-n-3}\right)=\mu_{p+1}^{-1}\left(C_{n+1}^{(p+1)}(n+2) r_{p, p+1}^{n}-D_{n+1}^{(p+1)}(n+1) r_{p, p+1}^{-n-3}\right) .
$$

Из условия инвариантности модуля векторного потенциала при переходе через границу раздела сред

$$
\left|\vec{A}^{(p)}\left(r_{p, p+1} ; \theta\right)\right|=\left|\vec{A}^{(p+1)}\left(r_{p, p+1} ; \theta\right)\right|
$$

с учётом выражений (1), (4), (9) получается вторая бесконечная подсистема, содержащая $n(N-1)$ алгебраических уравнений вида

$$
\begin{aligned}
& \left(C_{n+1}^{(p+1)}-C_{n+1}^{(p)}\right) r_{p, p+1}^{n+1}+\left(D_{n+1}^{(p+1)}-D_{n+1}^{(p)}\right) r_{p, p+1}^{-n-2}=\frac{(2 n+3)\left(\mu_{p}-\mu_{p+1}\right) I a^{2} r_{p, p+1}}{2(n+2)(n+1)} \sum_{s=0}^{\infty} \prod_{i=0}^{2 s} \frac{1}{2}+i \\
& \times \prod_{j=1}^{s+1} \frac{2 j-1}{2 j}\left(2 a r_{p, p+1}\right)^{2 s} \int_{0}^{\pi} \frac{\sin ^{2 s+2} \theta P_{n+1}(\cos \theta) d \theta}{\left(z_{0}^{2}+r_{p, p+1}^{2}-2 z_{0} r_{p, p+1} \cos \theta+a^{2}\right)^{2 s+3 / 2}} .
\end{aligned}
$$

Так как поле конечно при $r=0$, и неограниченно мало при $r \rightarrow \infty$, то подсистема (15) дополняется уравнениями: $D_{n+1}^{(1)}=0$ и $C_{n+1}^{(N)}=0$; здесь $p=1 ; p=N$ - индексы крайних внутренней и внешней сред магнитной системы.

Для случая расположения витка в экваториальной плоскости $z_{0}=0$ правая часть уравнений (15) примет следующий вид:

$$
\begin{aligned}
b=-\frac{\pi(2 n+3)\left(\mu_{p}-\mu_{p+1}\right) I a}{4 \sqrt{\pi}(n+2)(n+1) \sqrt{r_{p, p+1}^{2}+a^{2}}} \sum_{s=0}^{\infty} & \prod_{i=0}^{2 s} \frac{1}{2} \frac{1}{1+i} \prod_{j=1}^{s+1} \frac{2 j-1}{2 j}\left(\frac{2 a r_{p, p+1}}{r_{p, p+1}^{2}}\right)^{2 s+1}\left(\frac{1}{s+2}\right) \times \\
& \times \prod_{k=0}^{\infty}\left(1+\frac{n}{(s+3+k) 2}\right)\left(1-\frac{n}{(s+1+k) 2}\right)\left(1+\frac{n}{2(1+k)}\right)\left(1+\frac{n}{1-2 k}\right) .
\end{aligned}
$$

Таким образом, составляя систему уравнений из подсистемы (13) и подсистемы (15), получаем $2 n$ уравнений: $D_{n+1}^{1}=0$ и $C_{n+1}^{N}=0$. Принимая во внимание (16), можно определить постоянные $C$ и $D$, а, следовательно, магнитную индукцию поля искажения. Модуль результирующей индукции магнитного поля в любой точке магнитной системы определяется из выражения

$$
\left|\vec{B}^{(p)}\right|=\sqrt{\left(B_{r}^{(p)^{\prime}}+B_{r}^{(p)^{\prime \prime}}\right)^{2}+\left(B_{\theta}^{(p)^{\prime}}+B_{\theta}^{(p)^{\prime \prime}}\right)^{2}}
$$

Условие экстремума векторной функции записывается в виде $[16,17]$ :

$$
\frac{d \vec{B}}{d \vec{r}}=0
$$


где $\vec{r}$-радиус-вектор. Направление дифференцирования функции $\vec{B}$ осуществляется вдоль единичного вектора $\vec{i}_{r}$ для радиус-вектора. Производная по этому направлению определяется из выражения

$$
\frac{d \vec{B}}{d \vec{r}}=\left(\overrightarrow{i_{r}} \nabla\right) \vec{B}
$$

Так как в конкретном случае магнитное поле имеет вращательно-симметричную геометрию (3), с учётом [16] имеем

$$
\left(\vec{i}_{r} \nabla\right) \vec{B}=\frac{1}{2}\left[\operatorname{rot}\left(\vec{B} \times \vec{i}_{r}\right)+\operatorname{grad}\left(\vec{B} \vec{i}_{r}\right)-\vec{B}\left(\operatorname{div} \vec{i}_{r}\right)+\vec{i}_{r}(\operatorname{div} \vec{B})-\vec{B} \times\left(\operatorname{rot} \vec{i}_{r}\right)-\vec{i}_{r} \times(\operatorname{rot} \vec{B})\right]
$$

В силу соленоидальности магнитного поля $\operatorname{div} \vec{B}=0$, а так как, кроме того, $\operatorname{div} \vec{i}_{r}=0$, то $\operatorname{rot} \vec{i}_{r}=0$.

В данном случае

$$
\vec{B} \times \vec{i}_{r}=-B_{\theta} \vec{i}_{\varphi}, \quad \vec{B} \vec{i}_{r}=B_{r} .
$$

Тогда с учётом выражений (21) можно записать

$$
\frac{d \vec{B}}{d \vec{r}}=\frac{1}{2}\left[\operatorname{rot}\left(-B_{\theta} i_{\varphi}\right)+\mathrm{qrad} B_{r}-i_{r} \times(\operatorname{rot} B)\right] .
$$

В сферической системе координат с учётом выражения (3) и условия

$$
\frac{\partial B_{\theta}}{\partial \varphi}=\frac{\partial B_{r}}{\partial \varphi}=0
$$

можно записать

$$
\frac{d \vec{B}}{d \vec{r}}=\frac{\vec{i}_{r}}{2}\left[\frac{\partial B_{r}}{\partial r}-\frac{1}{r}\left(\frac{\partial B_{\theta}}{\partial \theta}+B_{\theta} \operatorname{ctg} \theta\right)\right]+\vec{i}_{\theta}\left(\frac{B_{\theta}}{r}+\frac{\partial B_{\theta}}{\partial r}\right) .
$$

Векторному уравнению (18) соответствует с учётом выражения (24) система двух скалярных алгебраических уравнений:

$$
\left\{\begin{array}{l}
\sin \theta\left(r \frac{\partial B_{r}}{\partial r}-\frac{\partial B_{\theta}}{\partial \theta}\right)-B_{\theta} \cos \theta=0 \\
B_{\theta}+r \frac{\partial B_{\theta}}{\partial r}=0
\end{array}\right.
$$

в которой частные производные от компонент вектора индукции магнитного поля для $p$-среды с учётом выражений (1), (2), (17) имеют вид:

$$
\begin{aligned}
& \frac{\partial B_{r}^{(p)^{\prime}}}{\partial r}=\frac{2 \mu_{p} I a^{2}}{\left(z_{0}^{2}+r^{2}-2 z_{0} r \cos \theta\right)^{0,5}} \sum_{n=0}^{\infty} \prod_{i=0}^{2 n} \frac{0,5+i}{1+i} \prod_{j=1}^{n+1} \frac{2 j-1}{2 j} \frac{(n+1)(2 a r \sin \theta)^{2 n}}{\left(z_{0}^{2}+r^{2}-2 z_{0} r \cos \theta+a^{2}\right)^{2 n+1,5}} \times \\
& \times\left\{\cos \theta+\left(r \cos \theta-z_{0}\right)\left[\frac{z_{0} \cos \theta-r}{z_{0}^{2}+r^{2}-2 z_{0} r \cos \theta}+\frac{2 n}{r}+\frac{(2 n+1,5)\left(2 z_{0} \cos \theta-2 r\right)}{z_{0}^{2}+r^{2}-2 z_{0} r \cos \theta+a^{2}}\right]\right\}, \\
& \frac{\partial B_{r}^{(p)^{\prime \prime}}}{\partial r}=\sum_{n=0}^{\infty}\left(C_{n+1}^{(p)} n r^{n-1}-D_{n+1}^{(p)}(n+3) r^{-n-4}\right)\left[(n+1) P_{n+2}^{1}(\cos \theta)-(n+1) \cos \theta P_{n+1}^{1}(\cos \theta)\right](\sin \theta)^{-1}, \\
& \frac{\partial B_{\theta}^{(p)^{\prime}}}{\partial \theta}=\frac{-\mu_{p} I a^{2} r \sin \theta}{\left(z_{0}^{2}+r^{2}-2 z_{0} r \cos \theta\right)^{0,5}} \sum_{n=0}^{\infty} \frac{(2 a r \sin \theta)^{2 n}}{\left(z_{0}^{2}+r^{2}-2 z_{0} r \cos \theta+a^{2}\right)^{2 n+2,5}} \prod_{i=0}^{2 n} \frac{0,5+i}{1+i} \prod_{j=1}^{n+1} \frac{2 j-1}{2 j} \times \\
& \times\left\{(2 n+1) 2 z_{0} r \sin \theta+\left[2(n+1) a^{2}-\left(z_{0}^{2}+r^{2}-2 z_{0} r \cos \theta\right)(2 n+1)\right]\left[\operatorname{ctg} \theta(1+2 n)-z_{0} r \sin \theta \times\right.\right. \\
& \left.\left.\times\left(\frac{1}{z_{0}^{2}+r^{2}-2 z_{0} r \cos \theta}+\frac{(2 n+2,5)^{2}}{z_{0}^{2}+r^{2}-2 z_{0} r \cos \theta+a^{2}}\right)\right]\right\},
\end{aligned}
$$




$$
\begin{gathered}
\frac{\partial B_{\theta}^{(p)^{\prime \prime}}}{\partial \theta}=-(\sin \theta)^{-1} \sum_{n=0}^{\infty}\left(C_{n+1}^{(p)} r^{n}(n+2)-D_{n+1}^{(p)} r^{-n-3}(n+)\right)\left[(n+1) P_{n+2}^{1}(\cos \theta)-(n+2) \cos \theta P_{n+1}^{1}(\cos \theta)\right], \\
\frac{\partial B_{\theta}^{(p)^{\prime}}}{\partial r}=\frac{-\mu_{p} I a^{2} r \sin \theta}{\left(z_{0}^{2}+r^{2}-2 z_{0} r \cos \theta\right)^{0,5}} \sum_{n=0}^{\infty} \prod_{i=0}^{2 n} \frac{0,5+i}{1+i} \prod_{j=1}^{n+1} \frac{2 j-1}{2 j} \frac{(2 a r \sin \theta)^{2 n}}{\left(z_{0}^{2}+r^{2}-2 z_{0} r \cos \theta+a^{2}\right)^{2 n+2,5}} \times \\
\times\left\{\left(2 z_{0} \cos \theta-2 r\right)(2 n+1)+\left[2(n+1) a^{2}-\left(z_{0}^{2}+r^{2}-2 z_{0} r \cos \theta\right)(2 n+1)\right] \times\right. \\
\left.\times\left[\frac{2 n+1}{r}+\frac{\left(z_{0} \cos \theta-r\right)}{z_{0}^{2}+r^{2}-2 z_{0} r \cos \theta}+\frac{(2 n+2,5)\left(2 z_{0} \cos \theta-2 r\right)}{z_{0}^{2}+r^{2}-2 z_{0} r \cos \theta+a^{2}}\right]\right\}, \\
\frac{\partial B_{\theta}^{(p)^{\prime \prime}}}{\partial r}=-\sum_{n=0}^{\infty}\left(C_{n+1}^{(p)} r^{n-1}(n+2) n+D_{n+1}^{(p)} r^{-n-4}(n+3)(n+1)\right) P_{n+1}^{1}(\cos \theta) .
\end{gathered}
$$

После подстановки (3) (6), (26)-(31) в (25) получим систему двух алгебраических уравнений с неизвестными $r$ и $\theta$ из которой определяются пары $r$ и $\theta$, являющиеся координатами точек, в которых индукция магнитного поля $B$ имеет экстремумы.

Таким образом, для оценки состояния магнитной системы $p$-среды получена зависимость в виде (25), которая позволяет определить индукцию магнитного поля только в той точке, где она имеет экстремальное значение.

Для случая, когда контур витка с током лежит в экваториальной $\left(z_{0}=0\right)$ плоскости магнитной системы (из физических соображений ясно, что точки максимума лежат в плоскости $\theta=\frac{\pi}{2}$ ), необходимо решить второе уравнение системы (25).

Для этого представим второе уравнение системы (25) в виде

$$
B_{\theta}^{\prime}+r \frac{\partial B_{\theta}^{\prime}}{\partial r}=-\left(B_{\theta}^{\prime \prime}+r \frac{\partial B_{\theta}^{\prime \prime}}{\partial r}\right) .
$$

Подставляя в формулу (32) выражения (6), (30), (31), после преобразований для $p$-среды получим

$$
\begin{aligned}
B_{\theta}^{\prime}+r \frac{\partial B_{\theta}^{\prime}}{\partial r}=- & \frac{\mu_{p} I a^{2}}{\left(r^{2}+a^{2}\right)^{2,5}} \sum_{n=0}^{\infty} \prod_{i=0}^{2 n} \frac{0,5+i}{1+i} \prod_{j=1}^{n+1} \frac{2 j-1}{2 j}\left(\frac{2 a r}{r^{2}+a^{2}}\right)^{2 n}\left[2 a^{2}(n+1)(1+2 n)-\right. \\
& \left.-r^{2}(2 n+1)(3+2 n)+\frac{2 r^{4}(2 n+1)(2 n+2,5)-4 a^{2} r^{2}(n+1)(2 n+2,5)}{r^{2}+a^{2}}\right], \\
& -\left(B_{\theta}^{\prime \prime}+r \frac{\partial B_{\theta}^{\prime \prime}}{\partial r}\right)=\sum_{n=0}^{\infty} P_{n+1}^{1}(\cos \theta)\left[C_{n+1}^{(p)}(n+1)(n+2) r^{n}+D_{n+1}^{(p)}(n+1)(n+2) r^{-n-3}\right]
\end{aligned}
$$

Согласно [19], правая часть выражения (34) представляет собой разложение в ряд по присоединенным функциям Лежандра левой части выражения (33). Такое разложение представляется в виде [19]:

$$
f(r ; \theta)=\sum_{n=0}^{\infty} a_{n+1} P_{n+1}^{1}(\cos \theta),
$$

где для коэффициентов разложения имеем

$$
a_{n+1}=C_{n+1}^{(p)}(n+2)(n+1) r^{n}+D_{n+1}^{(p)}(n+1)(n+2) r^{-n-3} .
$$

Из [19] известно, что любую регулярную функцию, определённую в интервале $(0, \pi)$, можно разложить в ряд по присоединённым функциям Лежандра:

$$
F(r ; \theta)=\sum_{n=0}^{\infty} A_{n+1} P_{n+1}^{1}(\cos \theta) .
$$


Так как функция, записанная в виде выражения, стоящего в левой части формулы (33), на основании (4) определена в интервале $(0, \pi)$, кроме точек, расположенных на контуре витка с током, разложим её в ряд (37). Согласно [19] коэффициенты разложения этой функции имеют вид

$$
A_{n+1}=\frac{2(n+1)+1}{2(n+1)(n+2)} \int_{0}^{\pi} F(\theta) P_{n+1}^{1}(\cos \theta) \sin \theta d \theta .
$$

Заменяя переменную $x=\cos \theta$ в выражениях (33), (38), немой индекс $n$ на $s$ в выражении (33) и подставляя правую часть выражения (33) вместо $F(r ; \theta)$ в фрормулу $(38)$, получаем

$$
\begin{gathered}
A_{n+1}=-\frac{(1+2(n+1)) \mu_{p} I a^{2}}{2(n+2)(n+1)\left(r^{2}+a^{2}\right)^{2,5}} \sum_{s=0}^{\infty} \prod_{i=0}^{2 s} \frac{0,5+i}{1+i} \prod_{j=1}^{s+1} \frac{2 j-1}{2 j}\left(\frac{2 a r_{p, p+1}}{r_{p, p+1}^{2}+a^{2}}\right)^{2 s}\left[2 a^{2}(s+1)(1+2 s)-\right. \\
\left.-r^{2}(2 s+1)(3+2 s)+\frac{2 r^{4}(2 s+1)(2 s+2,5)-4 a^{2} r^{2}(s+1)(2 s+2,5)}{r^{2}+a^{2}}\right] \int_{-1}^{1}\left(1-x^{2}\right)^{s+0,5} P_{n+1}^{1}(x) d x .
\end{gathered}
$$

Замена в подынтегральной части выражения (39) $\mu=1, \gamma=n+1, \lambda=s+1,5$ приводит этот интеграл к известному определённому интегралу [20]:

$$
\int_{-1}^{1}\left(1-x^{2}\right)^{\lambda-1} P_{\gamma}^{\mu}(x) d x=\frac{\pi 2^{\mu} \Gamma\left(\lambda+\frac{\mu}{2}\right) \Gamma\left(\lambda-\frac{\mu}{2}\right)}{\Gamma\left(\lambda+\frac{\gamma}{2}+1\right) \Gamma\left(\lambda-\frac{\gamma}{2}\right) \Gamma\left(1-\frac{\mu}{2}+\frac{\gamma}{2}\right) \Gamma\left(\frac{1}{2}-\frac{\mu}{2}-\frac{\gamma}{2}\right)},
$$

где $\lambda, \mu, \gamma$-комплексные параметры, причём $2 \operatorname{Re} \lambda>|\operatorname{Re} \mu|$, что справедливо для решаемой задачи; $\Gamma(s)$ - гамма функция.

Согласно (40), определённый интеграл, присутствующий в (39), преобразуется к виду

$$
L=\frac{2 \pi \Gamma(s+2) \Gamma(s+1)}{\Gamma\left(s+\frac{n}{2}+3\right) \Gamma\left(s+1-\frac{n}{2}\right) \Gamma\left(1+\frac{n}{2}\right) \Gamma\left(-\frac{n+1}{2}\right)} .
$$

Таким образом, если учесть выражения (35), (37), то левая и правая части формулы (32) являются разложением в ряд по присоединённым функциям Лежандра. Эти ряды будут равны в том случае, если соответствующие члены правого ряда будут равны членам левого ряда выражения (32). Применяя метод сравнения рядов и учитывая, что члены ряда (35) отличаются от членов ряда (37) только коэффициентами разложения, можно заключить, что ряд (35) будет равен ряду (37) в том случае, если соответствующие коэффициенты разложения (36) будут равны коэффициентам разложения (38). Условие равенства рядов имеет вид

$$
a_{n+1}=A_{n+1}
$$

где $n=0,1, \ldots, \infty$.

Поскольку для рассматриваемой модели геометрия электромагнитной системы, кроме осевой симметрии, приобретает ещё и симметрию относительно плоскости $\theta=\frac{\pi}{2}$, и $P_{n+1}^{1}(\cos \theta)=0$, где $n$-нечётное число, то в приведённых выше выражениях (34)-(39), (41) для магнитного поля необходимо заменить индекс $n+1$ на $2 n+1$ [20], оставив при этом индекс суммирования рядов без изменения. Приравнивая коэффициенты разложения согласно выражению (42) для всех членов ряда выражения (32), получим бесконечное число уравнений, содержащих только одно неизвестное $r$. Одинаковые корни для всех этих уравнений будут являться решением второго уравнения системы (25) и координатами точек, в которых вектор индукции магнитного поля имеет экстремальные значения.

Полученное аналитическое выражение в виде бесконечного числа уравнений с одним неизвестным может найти применение при автоматизированном проектировании аналогичных электромагнитных систем (в том числе, шаровых электродвигателей), так как позволяет с небольшими затратами времени на ЭВМ с высокой точностью определить координаты экстремумов вектора индукции магнитного поля в каждой из сред электромагнитной системы, если катушка обмотки представлена в виде системы софокусно расположенных в экваториальной плоскости круговых, бесконечно тонких витков с током, а также, если электромагнитная система 
представлена такими катушками, повёрнутыми друг относительно друга на некоторый угол. Зная координаты точек экстремумов, по приведённой математической модели магнитного поля, можно определить в них значения индукции магнитного поля в каждой из сред электромагнитной системы, что, в свою очередь, позволит спроектировать электромагнитную систему, экстремальные значения индукции которой находятся в заданных пределах.

\section{БИБЛИОГРАФИЧЕСКИЙ СПИСОК}

1. Дементъев, В.М. Вращательно-симметричное магнитное поле статического контурного тока в «сферическом» многосредном пространстве [Текст] / В. М. Дементьев, А. И. Скороспешкин / Электрические машины. Машинновентильные системы, коммутация коллекторных электрических машин: Межвузов. сб. научн. тр. - Куйбышев: КПтИ, 1978. - Вып. 4. - С. 65-72.

2. Фомин, А. А. Шар в поле витка с током [Текст] / А. А. Фомин // жТТ. - 1963. - Т. 33, Вып. 9. - С. 1021-1030.

3. Абламунеи, И. Г. Вращающийся шар в поле витка с током [Текст] / И. Г. Абламунец, О. П. Прудников // ЖТФ.1990. - Т. 60, Вып. 6. - С. 1-12.

4. Гримальский, О.В. Метод расчёта трёхмерного электромагнитного поля тонких пластин и оболочек [Текст] / О. В Гримальский // Извест. АН СССР. Энергетика и транспорт. - 1990. - № 6. - С. 61-68.

5. Апполонский, С. М. Расчёт неоднородной экранирующей сферической оболочки [Текст] / С. М. Апполонский // Изв. вузов. Электромеханика. - 1986. - № 10. - С. 110-113.

6. Петленко, Б.И. Оптимизация комбинированного электромагнитного экрана по массе [Текст] / Б. И. Петленко, А. Е. Дергачев // Электричество. - 1990. - № 11. - С. 62-65.

7. Лавров, В.Я. Применение принципа Гюгенса-Кирхгофа для идентификации электромагнитного поля в сферических координатах. [Текст] / В. Я. Лавров, А. В. Кирпанев, А. П. Пуханов // Техническая электродинамика. 1990. - № 6. - С. 18-22.

8. Замидра, А. И. Расчёт осесимметричного магнитного поля в системах со сферическим экраном [Текст] / А. И. Замидра, А. И. Трохименко // Техническая электродинамика. - 1984. - № 4. - С. 21-26.

9. Граве, В.И. Намагничивание шарового ферромагнитного слоя и сплошного ферромагнитного шара в магнитном поле кругового контура с током [Текст] / В. И. Граве // Теоретическая электротехника (Львов) - 1987. - № 43. C. $13-16$.

10. Ивлиев, Е.А. О синтезе магнитных полей в слоистых средах [Текст] / Е. А. Ивлиев, Ю. Я. Иосиль, Л. А. Цейтлин // Электричество. - 1989. - № 2. - С. 88-89.

11. Калимов, А.Г. Применение интегро-дифференциального уравнения магнитостатики для расчёта тонкостенных ферромагнитных экранов [Текст] / А. Г. Калимов // Электричество. - 1999. - № 11. - С. 36-38.

12. Одилов, Г. Представление решения краевой задачи для уравнения электромагнитного поля электрической машины в свёрнутой форме [Текст] / Г. Одилов, Э. Хакимов // Изв. АН УзССР. Сер. техн. наук. - 1985. - № 5. C. $16-21$.

13. Петров, С.И. Электромагнитное поле в сферической полости с вращающимся шаром [Текст] / С. И. Петров // Изв. вузов. Электромеханика. - 1991. - № 3. - С. 20-26.

14. Лютахин, Ю.И. Алгоритм определения максимумов индукции магнитного поля электромагнитной системы шарового электродвигателя [Текст] / Ю. И. Лютахин, В. М. Дементьев / Специальные электрические машины: Межвузов. сб. научных тр. - Куйбышев: КПтИ, 1983. - С. 36-40.

15. Лютахин, Ю.И. Оптимальное проектирование электромагнитной системы шарового электродвигателя [Текст] / Ю. И. Лютахин / Электрические машины специального назначения: Межвузов. сб. научных тр. - Куйбышев: КПтИ, 1985. - С. 12-19.

16. Корн, Г. Справочник по математике для научных работников и инженеров [Текст] / Г. Корн, Т. Корн.- М.: Наука, 1984. - 809 с.

17. Тамл, И.Е. Основы теории электричества [Текст] / И. Е. Тамм. - М.: Наука, 1976. -616 с.

18. Штафль, М. Электродинамические задачи в электрических машинах и трансформаторах [Текст] / М. Штафль. М.-Л: Энергия, 1966. - 200 с.

19. Шимони, К. Теоретическая электротехника [Текст] / К. Шимони. - М.: Мир, 1964. -773 с.

20. Градштейн, И.С. Таблицы интегралов, сумм, рядов, произведений. [Текст] / И. С. Градштейн, И. М. Рыжик. M.: Наука, 1971. - 638 с. 